\title{
Smiling and laughter: Different phyletic origins?
}

\author{
J. S. LOCKARD, C. E. FAHRENBRUCH, J. L. SMITH, and C. J. MORGAN \\ University of Washington, Seattle, Washington 98195
}

\begin{abstract}
Ethological data on 141 adult dyads support the hypothesis that the human smile had its origin in the silent bared-teeth submissive grimace of primates and that the facial expression accompanying laughter evolved from the relaxed open-mouth display of play. Affiliative smiling occurred in greeting and departure interactions, whereas frank laughter was almost exclusively seen in a recreational context. Convergence and learning may mask the original distinction, leaving the impression of a continuum of graded signals.
\end{abstract}

The ethological problem of whether laughter is simply a more intense form of smiling or whether the two displays have different phyletic origins is addressed in this study. The consensus in the past placed smiling and laughter on a simple continuum of graded intensities (Andrew, 1963; Hinde, 1974). More recently it has been suggested that the continuum is, at least, twodimensional, with affiliativeness of the facial expression on one axis and playfulness on the other (van Hooff, $1967,1971)$. In other words, it has been hypothesized that smiling and laughter were originally two different displays in nonhuman primates but have converged in humans: smiling having its origin in the silent baredteeth submissive grimace (Figure 1, left side) of primates and laughter evolving from the relaxed open-mouth display (Figure 1, right side) of play. These facial grimaces are distinguished primarily by the extent of teeth exposure, degree of lip retraction, and the curvature of the corners of the mouth. The two displays are usually distinctively different in those primate genera such as macaques (Macaca) and guenons (Cercopithecus) possessing both behaviors (van Hooff, 1967). In chimpanzees (Pan troglodytes) they are neither temporally nor categorically related (i.e., as determined by sequential and factor analysis, respectively) (van Hooff, 1971). In a few primate species, for example, drills (Mandrillus leucophaeus, Mandrillus sphinx) and gelada baboons (Theropithecus gelada), the forms of the displays are still different, but the contexts in which they occur are less distinct (van Hooff, 1967).

The present authors tentatively propose that, if smiling and laughter are converging, the process is incomplete and that evidence of the distinctiveness of the two expressions is still seen in humans. Although smiling and laughter may appear to shade into one another, an ethological study on children suggests that

Reprint requests should be addressed to the first author, Department of Psychology or Department of Neurological Surgery, University of Washington, Seattle, Washington 98195. laughter is factorially associated with "rough-andtumble" play, whereas smiling falls into a "social" cluster (Jones, 1972). If in adult interactions, as well, smiling and laughter do serve separate functions, their different facial manifestations should be reliably detectable. Therefore, the present study was undertaken to discern the differences, in both form and context, that adult smiling and the facial expression accompanying laughter may take. It was hypothesized that if such
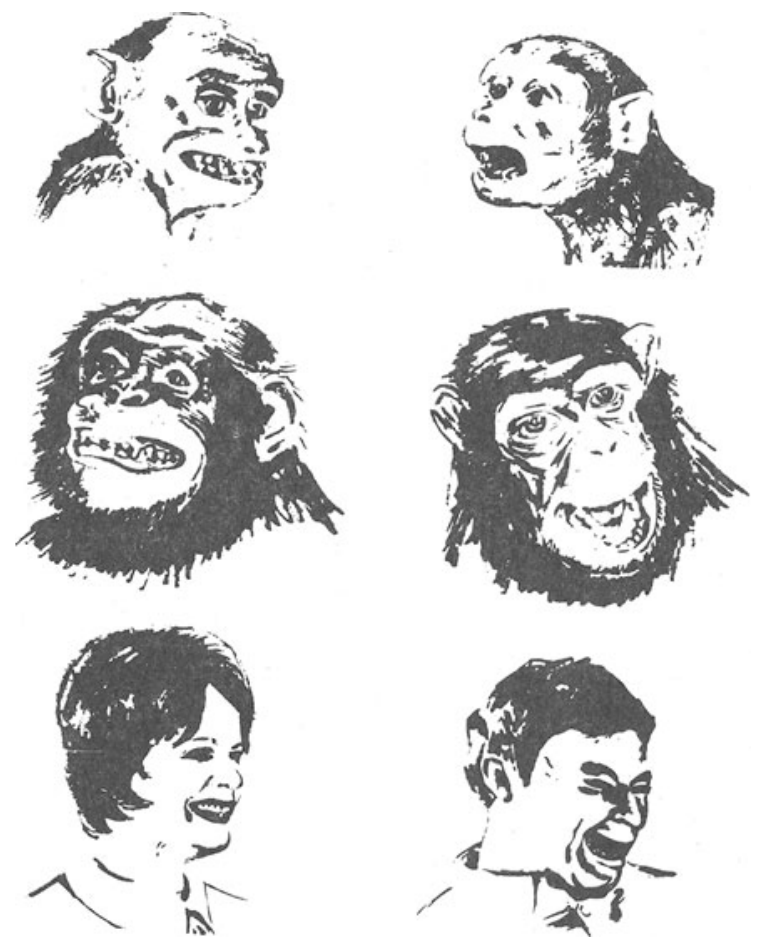

Figure 1. Sketches illustrating the possible origin of human smiling (left side) and laughter (right side). The silent bared-teeth display of submission and the relaxed open-mouth display of play are shown for the rhesus monkey (top) and chimpanzee (middle), suggestive in both form and context of the human smile and the facial expression accompanying laughter, respectively. (Sketches by Z. Zelenak.) 
displays were originally phyletically different and still serve to some extent their initial functions, then they should be either temporally distinct or mutually exclusive in certain types of social exchanges.

\section{METHOD}

The form, frequency, and temporal occurrence of smiling and laughter were observed and systematically recorded between adult dyads (pairs) in four social situations: (1) goal-oriented interactions (e.g., buying a meal or purchasing a ticket); (2) work breaks (e.g., at coffee or lunch time); (3) chance encounters (e.g., greeting exchanges in hallways at work or between college classes); and (4) leisure episodes (e.g., dining out or social gatherings).

Four intensities of smiling and laughter, respectively, were defined. Smiling ranged from closed lips, or front-teeth exposure, with sharp mouth corners (slightly turned up) to open mouth, teeth-biting edges showing (particularly lower jaw) and rounded mouth corners. The least intense smiles were similar to a bared-teeth display and the more intense smiles approximated a play-face expression. Laughter varied from a barely audible grunt (e.g., "hah") or mild chuckle with some vertical chest movement to an intense guffaw with both head and chest movements and sometimes eye closure. (The head moved backward and then forward, and the chest movements were very exaggerated in the most intense laughter category.) Frank laughter was typical of only the most intense vocalizations.

The initiating individual of 141 different dyadic interactions was observed from the beginning to the end of the exchange. The observations were divided in to 5 -sec intervals manifesting a mean interaction duration of $15.7,20.8,21.4$, and 16.2 intervals (corresponding to $78.5,104.0,107.0$, and $81.0 \mathrm{sec}$ ) for the goal, work, chance, and leisure situations, respectively. Four observers were employed, two females and two males. The interobserver reliability in recording the same dyads ranged from a correlation of .75 to .95 depending upon the particular smile or laughter category (i.e., the middle intensity categories were the least reliable).

\section{RESULTS}

In analyzing the data a numerical value of $1,2,3$, or 4 was assigned to smiles and laughter, respectively, depending upon the particular intensity of each (a value of 1 was least intensive and a value of 4 was most intensive). To evaluate the exclusiveness of the four social situations, mean values of smiling and laughter combined were subjected to an analysis of variance $(\mathrm{F}=20.65$, df $=3$ and $137, \mathrm{p}<.001)$. (First, the means of individual subjects were computed as the sum of their numerical values divided by the number of intervals, and then the group means were ascertained.) Student ts affirmed that the social situations were distinct from one another $(\mathrm{p}<.01$ to $\mathrm{p}<.001)$, with the individuals in the goal-oriented category manifesting the least intense smiles and laughter as compared to the leisure category, in which the smiling and laughter were more exaggerated (the work and chance situations were intermediate).

In analyzing laughter separately, Figure 2 shows that the most intense form of laughter (intensity 4) was almost exclusively restricted to the leisure category. Hearty chuckle-type vocalizations (intensity 3 ) were seen in both the chance and leisure interactions. Mild

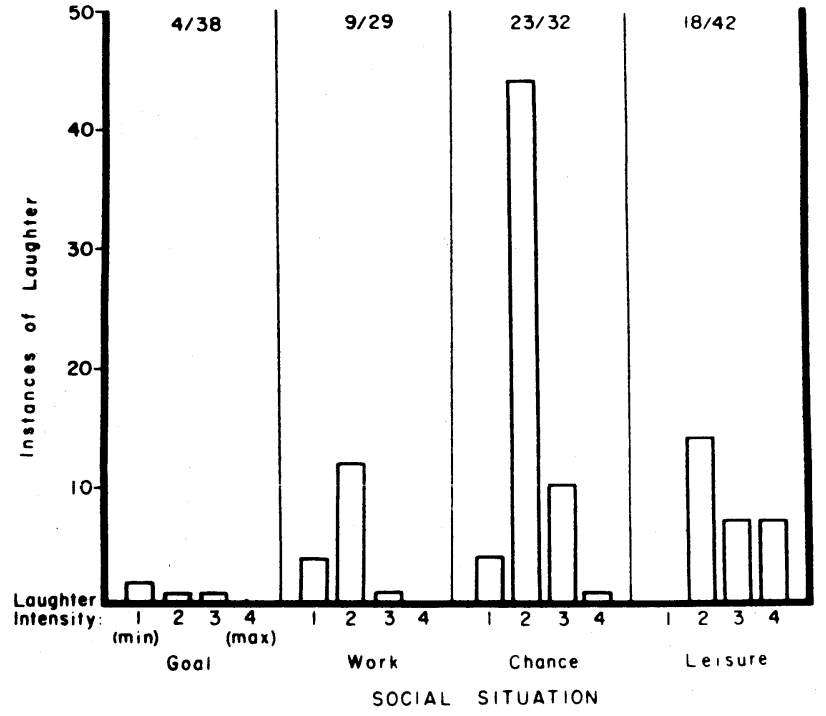

Figure 2. Instances of laughter, in terms of laughter intensity, are shown for four social situations (goal oriented, work break, chance encounter, and leisure episodes). The numbers at the top of the graph indicate the number of individuals, out of the total sample for each social situation, who contributed to the data; for example, only 4 of 38 individuals manifested laughter of any intensity in the goal-oriented situation. Laughter intensity 1 was absent in the leisure situation. Laughter intensity 2 was particularly evident in the chance encounter interactions. Laughter intensity 3 was rarely seen in the goal-oriented and work-break categories. Laughter intensity 4 was prevalent only in the leisure interactions.

laughter (intensity 2) occurred in the dyadic greeting exchanges of work, chance, and leisure, but particularly in chance encounters. As expected, laughter intensity 1 was not seen in the leisure situation and laughter of any intensity was rare in the goal-oriented social situation.

In comparing different smiles, Figure 3 is illustrative where the milder forms of smiling (intensities 1 and 2, approximating the bared-teeth display) are seen most often as the first instance of smiling in all four social categories. Smiling intensities 3 and 4 (approximating play-face expressions) are infrequently seen in the goal, work, and chance categories but do not occur relatively often in the leisure situation. This finding was supported by the considerably greater percentage of mild smiles (intensities 1 and 2) in the first two (not shown) and in the last two intervals (Figure 4), respectively, of the goal, work, and chance dyadic interactions. As predicted, only in the leisure situation does one find more intense smiles (play-face expressions) occurring either initially, during, or at the terminal intervals of an interaction.

\section{DISCUSSION}

Although individual differences and cultural shaping of adult expressions tend to mask consistencies, the present findings support the hypothesis that front-teeth smiling and the more open-mouth expression associated with laughter have possibly distinct phyletic origins and functions. Mild forms of smiling 
are evident in greeting and departure interactions (affiliative). The facial expression of frank laughter is seen almost exclusively in a recreational context (play). If frank laughter does occur in the other social situations, it is usually during the later stages (preterminal of the dyadic interactions when the exchanges are more relaxed or less formal).

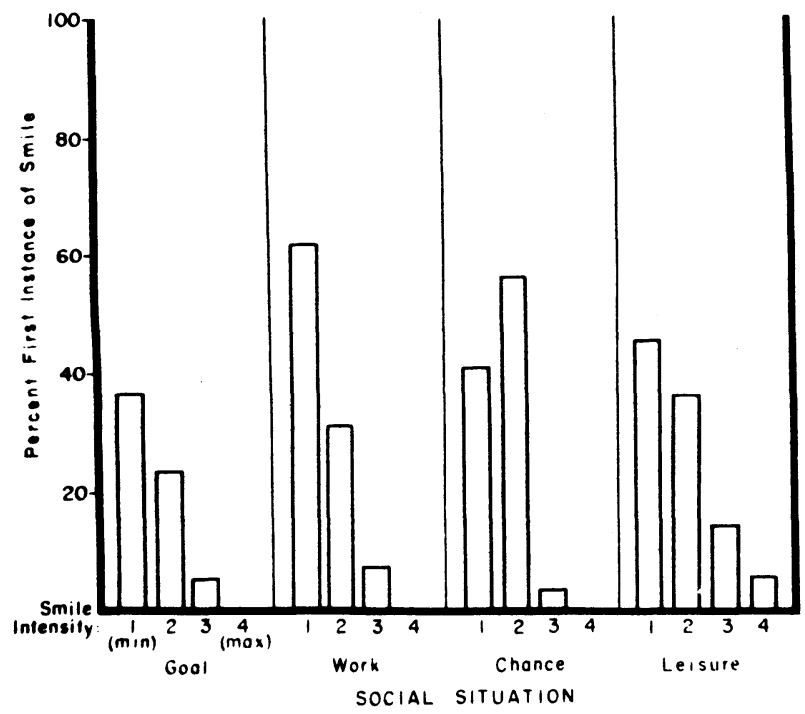

Figure 3. The smile intensity of the first instance of smile, in terms of percent of individuals, is shown for four different social situations (goal oriented, work break, chance encounter, and leisure episodes). Only in the leisure situation does the most intense smile (4, more intense play face) appear as the first smile of a dyadic interaction. Smile intensity 3 (less intense play face) is also more evident in the leisure category. Smile intensities 1 and 2 (front-teeth displays) frequently appear as the first instance of smiling in all four social situations.

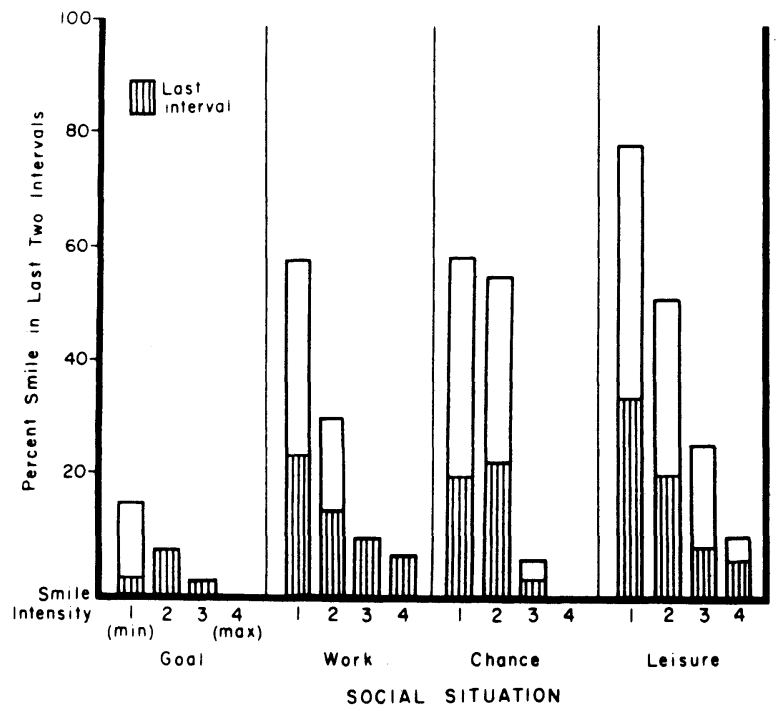

Figure 4. Percent individuals smiling in the last two intervals (the vertical-lined areas are data for the last interval only) of a dyadic interaction are given by smile intensity for four social situations (goal oriented, work break, chance encounter, and leisure episodes). Although infrequent in the goal-oriented situation, smile intensities 1 and 2 (front-teeth displays) are seen in the last two intervals of all four social situations. Smile intensities 3 and 4 (play-face displays) are seen as the last smile of a dyadic interaction most of ten in the leisure category.
There are some ontogenetic data in nonhuman primates and in humans to indicate that the play face is not a more intense form of the bared-teeth expression. In stump-tail macaques (Macaca arctoides) (Chevalier-Skolnikoff, 1974), the assumed precursor of the play face ("mutual mouth nibble") occurs developmentally either before or contiguously in time with the silent bared-teeth display ("grimace"). In humans, smiling and laughter are temporally separated in their development with little overlap. It is several months after birth before babies exhibit laughter (Sroufe \& Waters, 1976; Sroufe \& Wunsch, 1972). Although both the smile and laughter face may become with maturation more appropriately manifested (i.e., elicited by, and specific to, those social contexts in which adults would exhibit them), this development does not imply that the laughter face arises out of the smile face or that the former is a more intense form of the latter.

It is likely that the difficulty in detecting the facial differences between smiling and laughter in humans is, in part, a function of convergence of the two displays. If an initially submissive display such as the silent bared-teeth expression prevented agonistic encounters, it might well have come (through natural selection) to stand for affiliative intentions. Friendly signals have been of considerable importance in the survival of hominids and, therefore, might have been subject to ritualization. Exaggeration of affiliative smiling could have taken the form of auditory accentuation (e.g., vocalizations similar to laughter) or play-face simulation (e.g., expressions approximating the relaxed open-mouth display) or both. The latter possibility is supported by chimpanzee data in which one of three variants of the silent bared-teeth display, namely, an openmouth bared-teeth expression, is often reciprocally manifested in an affiliative interaction between two animals (van Hooff, 1971). In drills, gelada baboons and possibly two macaque species (Macaca nigra and Macaca maura) as well, the silent bared-teeth display may function either as a submissive or as a reassuring signal (van Hooff, 1967). These data have also been discussed in support of smiling sometimes serving as an invitation to approach in human mother-infant interactions (Vine, 1973).

Whereas auditory accentuation of affiliative smiling could occur through ritualization, learning is also a distinct possibility. If, as the data on children (Jones, 1972) suggest, the two displays tend to occur in different contexts, both of which signal friendly intentions, then it is conceivable that adults learn to mix the two displays. In the present data, laughter occurring in other than the leisure situation most frequently was of mild intensity (i.e., intensities 1 and 2), that which would be expected if interchanging of the two displays were socially advantageous. Moreover, in highly affiliative situations such as social reception lines and amicable interactions between superiors and subordinates, forced laughter is often apparent, consistent with an acquired blending of the two displays. Such variation in form and occurrence of smiling and laughter, either through convergence, learning, or both, would create the appearance of a continuum of graded signals concealing originally discrete displays of different phyletic origins.

\section{REFERENCES}

ANDREw, R. J. Evolution of facial expression. Science, 1963. 142, 1034-1041.

Chevalier-Skolnikoff, S. The ontogeny of communication in the stump-tail macaque (Macaca arctoides). In $\mathrm{H}$. Hofer, A. H. Schultz, \& D. Starch (Eds.), Contributions to primatology (Vol. 2). New York: S. Karger, 1974. Pp. 1-174.

HINDE, R. A. Biological basis of human social behaviour. New York: McGraw-Hill, 1974. Pp. 127-132.

Jones, N. B. (Ed.) Ethological studies of child behaviour. Cambridge: University Press, 1972. Pp. 97-127. 
Sroufe, L. A., \& WAters, E. The ontogenesis of smiling and laughter: A perspective on the organization of development in infancy. Psychological Review, 1976, 83, 173-189.

Sroufe, L. A., \& Wunsch, J. P. The development of laughter in the first year of life. Child Development. 1972. 43, 1326-1344.

van Hooff, J. A. R. A. M. The facial displays of the Catarrhine monkeys and apes. In D. Morris (Ed.). Primate ethology. London: Widenfeld \& Nicolson, 1967. Pp. 7-67. van Hooff, J. A. R. A. M. Aspects of the social behaviour and communication in human and higher non-human primates. Rotterdam: Bronder-Offset, 1971. Pp. 17-187.

VINE, I. In $M$. von Cranach \& 1 . Vine (Eds.), Social communication and movement. New York: Academic Press, 1973. Pp. 195-298.

(Received for publication May 9, 1977.) 